DOI: 10.14526/2070-4798-2019-14-3-17-22

\title{
Analysis of the main areas of research in archery abroad
}

\author{
Maksim V. Aranson*, Edvin S. Ozolin, Olga V. Tuponogova, Boris N. Shustin \\ Federal Scientific Center of Physical Culture and Sports \\ Moscow, Russia \\ ORCID: 0ooo-0oo2-5055-0997, aranson@yandex.ru* \\ ORCID: 0ooo-0001-6193-8893, edoz1002@mail.ru \\ ORCID: oooo-0002-7501-930X, olga.tuponogova@yandex.ru \\ ORCID: 0ooo-0002-5013-9912, shustin@vniifk.ru
}

\begin{abstract}
The study of foreign scientific research on various aspects of training athletes is necessary to determine the key areas of research teams work in the field of sports science. Research methods. Analysis of literature sources obtained from databases of scientific literature on archery for the period 2015-2019. Results. It was revealed that the largest number of studies were carried out on medical and biological topics and methods of training archers. Also of interest are the issues of organization, financing and historical aspects of the sport that influenced its development. Conclusion. When planning complex research in a particular sport and related sports, it is necessary first of all to pay attention to the issues that attract the close attention of of those countries scientific teams that have achieved significant success in these sports or in which they are intensively developing.
\end{abstract}

Keywords: archery, research, scientific directions, analysis.

For citation: Maksim V. Aranson*, Edvin S. Ozolin, Olga V. Tuponogova, Boris N. Shustin. Analysis of the main directions of research in archery abroad. Russian Journal of Physical Education and Sport. 2019; 14(3): 17-22. DOI: 10.14526/2070-4798-2019-14-3-17-22

\section{INTRODUCTION}

Archery has been part of the Olympic program since 1900 and is present (with a break during 19121972) until the present time. This sport is quite medal-intensive. It 5 sets of awards are played. In addition, world archery Championships and cups are constantly held. Recursive bows are used in the Olympic games, recursive and block bows are used in other major competitions. Archery is considered a technically challenging sport because it requires specific training.

In our country, researches are conducted on various aspects of the shooters training and its improvement, taking into account the modern requirements of sports activity [19]. However, despite the fact that the results of these studies are implemented in sports training, the Russian national team is not always among the winners of the largest competitions. To improve the effectiveness of training, it is necessary to make greater use of the foreign countries experience, especially those that achieve high results in the international arena.

The purpose of the work is to study the main directions of scientific research on the archers training abroad.

Research method: analysis of scientific and methodological literature (scientific articles, abstracts of articles, guidelines, etc.) on various aspects of training and providing training and competitive activities in archery.

\section{RESULTS AND DISCUSSION}

From the array of scientific materials on archery for the last 5 years (2015-2019), amounting to 6800 units, we selected 37 works for preliminary consideration, 19 were translated in the final version. The distribution of research by topic is shown in table 1. Basically, the research was conducted in the following areas: the development of inventory and equipment, including sports facilities; biomedical aspects (physiology, biochemistry, analysis of fitness indicators); psychological aspects; methods of training individual physical skills and capabilities; analysis of competitive activity and factors affecting performance; biomechanics of the shot and the characteristics of the arrow flight. A large number of patents have been registered on the equipment. Attention is drawn to the complete lack of work on the methods of recovery, including nutrition.

The considered works were performed by teams of researchers from countries that have reached a high level in archery (Korea, USA), or from countries in which this sport is intensively developing.

Research was carried out mainly by teams of educational institutions. There are works in which scientists from several institutions and even from different countries participated.

Here are the most interesting data obtained from the analysis of foreign sources.

An international team of researchers from the UK and Austria [2] studied the time phases of the 
archery cycle and identified key factors that affect the result. 14 potential predictors of the result were determined, and on their basis statistical modeling was carried out by the method of step multiple linear regression. The results of the study show that time to shot, release time, aiming time, arrow speed, and percentage of time from clicker to release account for $7.7 \%$ of the differences in the predicted outcome. The coach should focus on improving the relevant skills.

Table 1-Topics of foreign research on archery in 2015-2018

\begin{tabular}{|l|c|}
\hline \multicolumn{1}{|c|}{ Direction } & \% of published works \\
\hline Inventory, equipment and sports facilities & $21,6 \%$ \\
\hline Sports Physiology and Biochemistry & $16,7 \%$ \\
\hline The Psychology of Sports & $14,7 \%$ \\
\hline Other (history, development, sociology, etc.) & $14,7 \%$ \\
\hline General issues of building a training process & $12,7 \%$ \\
\hline Competitive activity & $8,8 \%$ \\
\hline Biomechanics of sport & $7,8 \%$ \\
\hline Sports selection and youth sport & $5,9 \%$ \\
\hline Injury and morbidity & $3,9 \%$ \\
\hline Management, finance & $0,9 \%$ \\
\hline Restoring and optimizing athletes' performance & - \\
\hline
\end{tabular}

Archery is a typical sport with "closed skills", it is characterized by repetition of movements worked out to automatism with maximum accuracy. The aim of the work of Italian researchers [18] is to assess the impact of the motor representations practice (mental visualization of movements without the presence of movements themselves) on the training process. The subjects performed the appropriate exercises, and then the experts evaluated the video on the following parameters: stance, tension and aiming, release and subsequent procedures (every 4 months, on 4 levels of descriptors). The work involved 2 groups of 10 people. The experimental group was shown videos of performances by members of the Italian national archery team. Discovered that in the experimental group improved results are expressed more significantly - the difference was $9 \%$, in the final stages of $3.2 \%$ in mid $-9,6 \%$; the control group showed improvement by an average $3.2 \%$. Training of motor representations promotes improvement of physical and mental possibilities of athletes, increase of efficiency and decrease in number of mistakes.

Korean researchers [7] studied factors affecting performance in archery and their relative importance Data for processing were obtained from a review of the literature on performance in archery; key performance factors were identified by a survey of experts (20 people), factor analysis (463 archers) and hierarchical analysis (36 specialists). Performance factors are divided into mental, skills (technical capabilities) and physical fitness factors.
The factors identified by the researchers and their relative importance can be used to build an optimal training in archery.

Skilled archers are more able to control the position of the body immediately after the release of the arrow than beginners. Australian researchers [3] studied the effect of training conditions on body oscillation in archers when standing in place. Subjects fired at a standard target in two modes: standing on the ground and standing on a raised platform. When shooting from a platform, body vibrations in the front-rear direction are much stronger. Therefore, during daily training athletes need to simulate the situation at the competition.

The result in archery may decline for various reasons, but data on their relative importance is not enough. Australian scientists [12] analyzed the results of shooting and grouping of arrows at the two largest international competitions in 2013. It is found that many archers lose points due to inaccurate adaptation of vision. In addition, as the level of the athlete increases, the relative importance of factors related to equipment also increases; the loss of points increases faster than the distance increases.

The aim of the work of Turkish scientists [14] is to study the activation strategy of 9 muscles of the forearm and shoulder girdle in archers of different levels of preparedness. Statistically significant differences between groups of athletes were found. It is shown that archers of the highest qualification use less distal muscles (forearm) and more 
proximal (shoulder) and axial (trapezoidal) for pulling the bowstring, while middle - level athletes and beginners use more distal muscles. To reduce horizontal bowstring vibrations to a minimum, archers need to load the axial and proximal muscles. The obtained data can be used to build an effective training of archers with the use of audiovisual feedback.

It is believed that when shooting outdoors, archers face obstacles that can affect their performance. However, there is insufficient literature on the various conditions that occur during shooting with regard to archery technique and its characteristics. The aim of the scientists from Malaysia study [9] is to study the shooting of an Archer in the open air under the influence of interfering factors using two shooting techniques: angular and linear. The experiment was conducted on one athlete in nine different conditions: morning, noon, night, heat, rain, windless, windy, cloudy and in extreme- 6 shots in 2 minutes. The results were processed in the Archery Score Pro program to determine the shooting characteristics. Classification of shooting methods was carried out according to the random tree algorithm. The classification analysis showed 83.3\% distinctiveness by shooting conditions; $97.9 \%$ of shots in extreme conditions were accurately classified, 98.1\% for the first three and last three shots. The results showed that the angular firing technique is superior to linear under different environmental conditions.

It is well known that exercises with elastic bands, as well as with their own weight in archery can significantly improve the technique of shooting, because they allow to simulate the movements when shooting without a bow and arrow. It is shown that the performance and technique of athletes significantly improve after the training program aimed at improving balance (exercises performed on an unstable support). However, these results are not obtained during official competitions. Italian researchers [10] compared the effect of three training regimes on the performance of highly skilled archers during official competitions. Participants performance was recorded during the competition immediately before and after the 6-month training program. All groups underwent specialized training (3 times a week for 90 minutes). In addition, KG-2 was trained on strength simulators, and the experimental one trained on the program, including work with elastic bands, calisthenics and exercises on BOSU balls with simulated archery. There were no statistically significant differences in performance between the three groups prior to the experiment, but the gains were statistically significantly different $(\mathrm{p}<0.05)$. In the experimental group, the result increased significantly more $(2.86 \pm 1.05 \%)$ than in KG-1 (1.04 $\pm 1.49 \%)$ and KG-2 $(0.15 \pm 1.22 \%)$. The use of unstable support and elastic bands to simulate archery techniques allows for more effective performance improvement of athletes in competitions compared to both a specific technical training program and a non-specific strength training program.

The aim of the work of Korean scientists [13] is to study the relationship between the characteristics of aiming and results in archery. Aiming characteristics were determined by averaging acceleration data during the aiming phase obtained from nine accelerometers in different parts of the body. A regression model was constructed for all subjects based on dynamic time distortion. The significance levels of the model were as high as $0.12 \%, 1.61 \%, 0.55 \%$, and $0.4 \%$, respectively. The developed regression model accurately describes the relationship between the characteristics of aiming and the result; it allows to find effective ways to improve performance in archery.

Experienced archers, unlike beginners, can quickly achieve a stable body position. The work of the international team [16] studied the effectiveness of training the main muscles that support the position of the body, to reduce the fluctuations of posture in teenage archers. In the experimental group, in addition to the usual training, an additional training program was carried out to strengthen the main muscle groups for a duration of 8 weeks. Data were collected before the experiment, 4 weeks after the start and at the end. Posture oscillations were measured in the anterior-posterior and lateral directions by the deviation of the pressure center. Factor analysis revealed a significant interaction in time between the result in pull-UPS, push-UPS, shooting performance and posture fluctuations. The amplitude of the pressure center fluctuation differed significantly between the experimental and control groups 4 and 8 weeks after the beginning of the experiment: in the experimental group, body oscillations on both axes were significantly less pronounced. It is obvious that reducing the amplitude of the body vibrations contributes to increased efficiency. It is recommended to introduce training of the main muscles that ensure the stability of the body into the training program of teenagers engaged in archery.

The aim of the Greek scholar's study [1] was to study age, competitive skills, and gender differences in psychological skills among Greek highly skilled archery athletes. 70 athletes completed the Greek version of the questionnaire "coping Skill in athletes-28" during the 2011 Greek national archery Championships for men and women. Differences between age groups on two factors (freedom from cares and peak pressure) are revealed. In addition, there were statistically significant differences between the groups on three factors (freedom from worries, confidence and concentration), but not between the sexes. Older athletes had better control 
of emotions and less stress.

In the work of Australian scientists [6] the relationship between the place occupied by the archery team in the qualifying round and the probability of winning prizes in the knockout matches is considered. The team's result in qualifying was used to determine the ability of each individual Archer in matches and calculate the probability of the team winning enough matches to achieve victory. It is found that the probability of a team winning the competition decreases exponentially depending on the qualification rating. These data adequately reflect the results of all major international archery competitions for 2000-2015. in order to have a chance of a medal, a team must take a place in the top four in the qualification round. The distribution of results for fourth place indicates the likely result values needed to win medals at major competitions.

Scientists from Australia [15] quantified the combined effect of body stability parameters before and after arrow release, tension force, arrow flight time, arrow length, and clicker response time on the performance or number of points scored by highly skilled archers shooting recurve bows. 39 archers from 4 different countries participated before participating in the archery world Cup. It was revealed that the decrease in reaction time, increase in the tension force and decrease in the rate of oscillations of the body are associated with the possibility of achieving better results.

American scientists [8] determined the effect of fatigue and increased heart rate on performance in archery with single and double Cam. To achieve fatigue, the athlete before shooting walked on a treadmill at an angle of $10^{\circ}$ at a speed of $4 \mathrm{~km} /$ $\mathrm{h}$ for 5 minutes, then 30 seconds rested and ran at $274 \mathrm{~m}$ at a speed of $11.2 \mathrm{~km} / \mathrm{h}$ and an angle of inclination of $1^{\circ}$. After the load, the athlete fired 4 shots at a distance of $18.28 \mathrm{~m}$ with a maximum speed, but without compromising accuracy. During all tests, the heart rate was continuously recorded. The aiming time did not differ significantly between double-Cam and single-Cam bows under both conditions. The accuracy of the first shot without fatigue and the third with fatigue did not differ significantly between the double and single-Cam bows. There was a weak relationship between heart rate and the accuracy of the first shot in a tired state for a single-Cam and double-Cam bow. Increasing the heart rate does not impair the execution time or accuracy of the shot when firing block bows with a single or double Cam.

"Calm eye" - a period of extended fixation of the gaze on the target-is observed in many tasks that require accurate aiming. It is known that in experienced athletes it is longer compared to that of beginners. The cognitive mechanisms underlying this phenomenon are not yet fully understood.
Scientists from the UK and the US [5] studied the duration of the "calm eye" period in experienced and novice archers in the field and in the laboratory using a computer task that simulates archery. The computer version also introduced random "noise" (visual interference). The control group consisted of people who are not involved in the shooting. In field and computer tasks, eye movements were measured using electro-oculography. Experienced archers a period of "calm eyes" in the field the job was longer compared with that for the beginners. In the computer task, archers also demonstrated a longer period of "calm eye" and greater accuracy compared to non-archers. In addition, experienced archers had an earlier onset and longer duration of "calm eye" in the presence of noise compared to beginners and non-archers. The longer duration of the "calm eye" may contribute to more effective programming and control of the athlete's actions.

Indonesian researchers [17] discuss the basic concepts associated with decisions in the process of choosing a sports archery business project, which over time can provide economic and social benefits. The construction of sports facilities for archery in Medan was considered. A feasibility study for the creation of an open shooting range has been prepared. The feasibility studies should take into account aspects related to the market and marketing, technology, equipment, management, legal issues, Economics, as well as social and financial aspects. Financial analysis using equity allowed to estimate the payback period, net present value and internal rate of return for this project.

The article of Korean scientists [11] is devoted to the study of the key political factors that led to the success of South Korea in archery. Based on a review of the Korean archery history, the authors included in the analytical framework of this study four key policy elements on green and Hoolihan and two additional key elements relating to the organization and management of high-performance sports. Policy aspects that have determined the success of archery development in South Korea: the establishment of the Korean archery Association (KAA) and the involvement of the business elite; increasing the number of archers able to engage in sport as a primary activity through the identification and support of young talented athletes; advances in sports science, training, equipment and sports facilities; providing more systematic opportunities for top-level archers to compete. Thus, the success of the Korean archers can be closely linked to the result of Kaa's strategy and policy initiatives with an eye to dominating the international sports movement.

The success of a country in a particular sport may be partly due to the fact that this type of physical activity is traditional for it. Korean researchers [4] considered the influence of traditional Korean 
archery (gukgung) on the development of archery in the country. It is noted that the ancient technique of shooting has much in common with the modern and it contributed to the formation of sports archery in Korea.

\section{CONCLUSION}

The identified areas of scientific research abroad reflect those aspects of training and competition activities and its provision, which have the greatest impact on the performance of athletes. Therefore, when planning complex research in this sport and related sports, it is necessary first of all to pay attention to the issues that attract the attention of scientific teams of those countries that have achieved significant success in these sports, or those in which they are intensively developing.

\section{REFERENCES}

1. Bebetsos E. Psychological skills of elite archery athletes. J. Hum. Sport Exerc. 2015; 10(2): $623-628$.

2. Callaway A.J., Wiedlack J., Heller M. Identification of temporal factors related to shot performance for indoor Recurve archery. Journal of Sports Sciences. 2017; 35(12):1142-1147.

3. Chalkley D., Giblin G., Pepping G.-J. Targeting Rio: Enhancing the Daily Training Environment in Archery Using Technology. Journal of fitness research. 2016; 5(Special Issue ASTN-Q Conference): 43-46.

4. Choi S., Ok. G. The Role of Gukgung in the Success of South Korean Archery. The International Journal of the History of Sport. 2016; 33(9): 9901007.

5. Gonzalez C.C., Causer J., Grey G.W., Miall R.C., Williams A.M. Exploring the quiet eye in archery using field and laboratory-based tasks. Exp Brain Res. 2017; 235: 2843-2855.

6. Jacobs D.B., Park J.L. Winning the team event at major international archery competitions. Proceedings of the Institution of Mechanical Engineers, Part P: Journal of Sports Engineering and Technology. 2017; 231(4): 355-361.

7. Kim H.-B., Kim S.-H., So W.-Y. The relative importance of performance factors in Korean archery. J Strength Cond Res. 2015; 29(5): 2111219.

8. Lankford D.E., Higginson B. The effect of fatigue and elevated heart rate on archery shot performance. International Journal of Exercise Science: Conference Proceedings. 2016; 8(4). URL: https://digitalcommons.wku.edu/ijesab/vol8/ iss4/59 доступ 23.01.2019

9. Loh W.P., Chong Y.Y. Classifying the Archery Performance with Conditional Effects on Angular and Linear Shooting Techniques. Journal of Telecommunication, Electronic and Computer
Engineering. 2018; 10(3-2): 95-99.

10. Monzoni R., Lucerini C.F., Federici A. Functional training is effective in improving archery sport performance. URL: //http://www.gnc. comwww.gnc.comwww.sportsci.orgwww.sportsci. org/2016/WCPASabstracts/ID-1605.pdf.

11. Park J.-W., Tan T.-C., Park H.-U. Interrogating the Key Policy Factors Behind South Korea's Archery Success. The International Journal of the History of Sport. 2016; 33(5): 523-544.

12. Park J.L., Layven J.E. Analysis of scores and arrow grouping at major international archery competitions. Proc IMechE, Part P: J Sports Engineering and Technology. 2014; 1-9. DOI: 10.1177/1754337113519760.

13. Quan C.H., Lee S. Relationship between Aiming Patterns and Scores in Archery Shooting. Korean Journal of Sport Biomechanics. 2016; 26(4): 353-360.

14. Simsek D., Cerrah A.O., Ertan H., Soylu R.A. Muscular coordination of movements associated with arrow release in archery. South African Journal for Research in Sport, Physical Education and Recreation. 2018; 40(1): 141-155.

15. Spratford W., Campbell R. Postural stability, clicker reaction time and bow draw force predict performance in elite recurve archery. European Journal of Sport Science. 2017; 17(5): 539-545.

16. Suppiah P.K., Kiet T.W.K., Musa R.M., Abdullah M.R., Lee J.L.F., Maliki A.B.H.M. The effectiveness of a core muscles stability program in reducing the postural sway of adolescent archers: a panacea for a better archery performance. International Journal of Physiotherapy. 2017; 4(5): 296-301.

17. Tarigan U.P.P., Mardhatillah A., Budiman I., Sembiring A.C., Ramadhan M.S. Feasibility Study Analysis of Establishing Archery Sports Special Place. IOP Conf. Series: Journal of Physics: Conf. Series. 2018; 1007 012027. doi :10.1088/17426596/1007/1/012027.

18. Tursi D., Napolitano S. Technical movements in archery. J. Hum. Sport Exerc. 2014; 9(Proc1): S570-S575.

19. Tarasova, L.V., Sabirova L.V., Tarasov P.Yu., Furaev V.D., Pankov V.A. Software of sports training in shooting sports. Vestnik sportivnoj nauki. 2018; 6: 14-17 (In Russ.).

20. Alexsandr S. Kuznetsov. Russian Professor's meeting. Pedagogiko-psihologicheskie I medicobiologicheskie problemy fizicheskoj kul'tury I sporta $=$ Russian Journal of Physical Education and Sport. 2019, 14(1), pp. 17-22. DOI: 10.14526/2070-47982019-14-1-18-24 (In Russ., In Engl.). 


\section{Submitted: 20.08.2019}

Author's information:

Maksim V. Aranson - Candidate of Biological Sciences, Federal Scientific Center of Physical Culture and Sport, 105005, Russia, Moscow, Elizavetinskiy per., House 10 Block 1, e-mail: aranson@vniifk.ru

Edvin S. Ozolin - Candidates of Pedagogics, Associate Professor, Federal Scientific Center of Physical Culture and Sport, 105005, Russia, Moscow, Elizavetinskiy per., House 10 Block 1, e-mail: edoz1002@mail. $\mathrm{ru}$

Olga V. Tuponogova - Candidates of Pedagogics, Associate Professor, Federal Scientific Center of Physical Culture and Sport, 105005, Russia, Moscow, Elizavetinskiy per., House 10 Block 1, e-mail: olga. tuponogova@yandex.ru

Boris N. Shustin - Doctor of Pedagogics, Professor, Federal Scientific Center of Physical Culture and Sport, 105005, Russia, Moscow, Elizavetinskiy per., House 10 Block 1, e-mail: shustin@vniifk.ru 